\title{
Risky Multicriteria Group Decision Making Based on Cloud Prospect Theory and Regret Feedback
}

\author{
Yan Song, ${ }^{1}$ Hao Yao, ${ }^{1}$ Shuang Yao, ${ }^{1}$ Donghua $Y u,{ }^{2}$ and Yan Shen ${ }^{3}$ \\ ${ }^{1}$ School of Economics and Management, Harbin Engineering University, No. 145, Nantong Street, \\ Nangang District, Harbin 150001, China \\ ${ }^{2}$ School of Computer Science and Technology, Harbin Institute of Technology, No. 92, Xidazhi Street, \\ Nangang District, Harbin 150001, China \\ ${ }^{3}$ College of Science, Harbin Engineering University, No. 145, Nantong Street, Nangang District, Harbin 150001, China \\ Correspondence should be addressed to Shuang Yao; alloniam@163.com
}

Received 29 December 2016; Revised 12 April 2017; Accepted 26 April 2017; Published 25 May 2017

Academic Editor: M. L. R. Varela

Copyright (C) 2017 Yan Song et al. This is an open access article distributed under the Creative Commons Attribution License, which permits unrestricted use, distribution, and reproduction in any medium, provided the original work is properly cited.

\begin{abstract}
The assessment of risky linguistic variables has significant applications in multiattribute group decision problems. This paper focuses on risky multicriteria group decision making using linguistic variable assessment and proposes a new model which considers various and differential psychological behavior and the ambiguity of linguistic variable assessment across multicriteria risks. Based on the cloud prospect value assessment, this paper proposes a cloud prospect value aggregation method and consensus degree measurement. An improved feedback adjustment mechanism based on regret theory is employed as the consistency model, which complements prospect theory. The three theoretical methods together constitute the core elements of the proposed CPD (cloud prospect value consensus degree decision) model. The feasibility and validity of the new decision making model are demonstrated with a numerical example, and feedback performance was compared with conventional direct feedback. The proposed CPD approach satisfies given consistency threshold of 0.95 and 0.98 after three and four feedback loops, respectively. Compared to the proposed CPD method, direct feedback approach needs seven and ten feedback loops under the same threshold, respectively, which shows that the proposed model increases efficiency and accuracy of group decision making and significantly reduces time cost.
\end{abstract}

\section{Introduction}

Multiple criteria decision making (MCDM) is an important part of modern decision science $[1,2]$. Group decision making under multicriteria risk refers to decision problems whose criteria are random variables; that is, the criteria values change with the uncertain environment $[3,4]$. The uncertainty is considered as risk under various states whose probabilities are known or measurable [5].

Many real-life decision making problems, such as investments, are group decision problems with multicriteria risk, often with a variety of uncertain factors and multiple uncertain states corresponding to multiple probabilities. Stock investment selection and decision making do not have exact attribute values due to the complex and uncertain environment with vagueness, ambiguity, and randomness.
Due to the complexity of the limited knowledge and decision maker (DM) perceptions, many alternative rankings occur for the uncertainty and randomness of the attribute value(s) [6]. According to their experience or related knowledge, DMs conduct fuzzy linguistic evaluations, such as "very poor," "poor," "fair," "good," and "very good." Prospect theory [7] considers DM psychological factors, which effectively corrects their maximum subjective expected utility, and has been successfully applied to individual $[8,9]$ and group [10-12] decision making. Therefore, to find the most desirable alternative or rank feasible alternatives to support decision making, it is critical to transform uncertain linguistic assessment, which mainly includes crisp or fuzzy numbers and their deformation.

The traditional technique for order preference by similarity to an ideal solution (TOPSIS) proposed by Hwang and 
Yoon [13] is a widely used crisp number method for classical MCDM. The TOPSIS method determines a solution with the shortest distance from the ideal solution and the farthest distance from the negative-ideal solution. Similar to TOPSIS, the VIKOR method proposed by Opricovic and Tzeng [14] considers the degree of closeness to the ideal solution using an integration function based on close to ideal solution. However, neither TOPSIS nor VIKOR considers the relative importance of the distance between two different reference points.

Fuzzy numbers, derived from fuzzy set theory, have become a main stream method to represent and handle uncertain attributes [15-18]. Criteria values include interval valued fuzzy numbers (IVFNs) [19] and the combination of the interval numbers fuzzy set and soft set [20]. The fuzzy set is well suited to dealing with vagueness [21]; for example, the hesitant fuzzy soft set based on soft and hesitant fuzzy sets has been successfully applied to group decisions [22].

The cloud theory is the innovation and development of a membership function for fuzzy set theory [23]. It transforms the uncertainty qualitative language concept into quantitative values [24] and has been successfully applied to data mining $[25,26]$, intelligent control, and intelligent algorithm modification [27, 28].

The different knowledge and evidence available to DMs result in inconsistent evaluations. To obtain the final group opinion, DMs should coordinate inconsistency in the group decision making process. An appropriate technique is to aggregate all the individual fuzzy preference relations and consistency processing $[29,30]$, repeatedly modifying the decision matrix.

Regarding the linguistic variable group decision problem, DMs usually participate in the decision making process and provide subjective evaluation in three different stages. The initial decision matrix is constructed in first stage, and the criteria weights are determined and incorporated in second stage. If the initial decision matrix fails the consistency check, the decision making process turns to the third stage and the initial decision matrix is reconstructed by the DMs. This process leads to multiple repeated subjective evaluation and increases computational complexity.

Although some current methods deal with linguistic variable assessment and provide feedback adjustment to some extent, some drawbacks remain. On one hand, current linguistic variable evaluation can only be applied to real numbers or triangular fuzzy numbers, which limits its application to other decision methods [31]. When prospect theory or related methods cannot be used directly [32], a transformation from linguistic variables to number values is required. On the other hand, feedback adjustment gives DMs little useful guidance to modify the decision matrix when the group evaluation does not satisfy the consistency threshold [33]. This modification process is time-consuming and blind, particularly for risky multicriteria group decision making. The number of DM modifications can be reduced by automatic negotiation, but this can lead to deviation from the DMs original intention to reach a consensus [22]. In addition, most group decision methods handle a single state rather than multiple states.
Group decisions based on linguistic variables are widespread in practice. Although the linguistic variables are processed through triangular fuzzy numbers or fuzzy logic, the variables are in a single state [11], not the multistate, which is not risky. For example, Wang and Lee [34] generalize TOPSIS to fuzzy multiple idea group decision making in fuzzy environment with single state criteria.

The cloud model introduced in [29] transforms the uncertainty relationship from qualitative (linguistic variable) to quantitative, accommodating fuzziness and randomness of qualitative evaluation. The model integrated two domains and constructed a mapping relationship between them. The cloud model is a continuous linguistic variable method [35] that has been widely used for multicriteria group decision making [36-38].

Therefore, we propose a method based on combining the cloud model and prospect theory, which integrates the advantages of vagueness and randomness from the cloud model and risk perception from prospect theory. The proposed model incorporates the cloud prospect value aggregation method and consensus degree measurement. An improved feedback adjustment mechanism provides the consistency model. Following Peng and Yang [19], we take full advantage of the nonexpected utility theory of regret-rejoice to compensate for the prospect theory. The new feedback adjustment rules handle DM inconsistency efficiently.

This paper proposes a new group decision making method that integrates the cloud model, prospect theory, and regret theory for risky multicriteria group decision making. Focusing on the group decision problem of multicriteria risky linguistic variables, we build a method for the cloud prospect consensus degree of risky multicriteria group decision making and feedback adjustment regulation. First, we use the cloud model to convert the uncertain linguistic variable problem to a risky multicriteria decision problem. The comprehensive prospect value is calculated, and the cloud prospect decision matrix of all DMs for all alternatives is constructed based on prospect theory. Then the decision matrixes for all DMs are aggregated and their consistency is measured. Those criteria scoring less than the acceptable threshold are returned to the DMs with guidance correction information according to the least regret value, and DMs modify their decision matrix with this correction information. Finally, the method sorts the alternatives.

\section{Problem Description and Theoretical Basis}

Consider the group decision problem of multicriteria risk with the alternatives sets $A=\left\{a_{1}, a_{2}, \ldots, a_{m}\right\}, m \geq 2$, and the associated criteria sets $Q=\left\{q_{1}, q_{2}, \ldots, q_{n}\right\}, n>2$, where each criterion is mutually independent. The weight vector of the criteria layer is $\omega=\left\{\omega_{1}, \omega_{2}, \ldots, \omega_{n}\right\}$, which satisfies the constraint $\sum_{i=1}^{n} \omega_{i}=1, \omega_{i} \geq 0, i=1,2, \ldots, n$. The possible natural states $f^{j}=\left\{f_{1}^{j}, f_{2}^{j}, \ldots, f_{s}^{j}\right\}$ of criteria $u_{j}$ have probability $p_{l}^{j}$ under state $f_{l}^{j}(1 \leq l \leq s)$. For the set of DMs $E=\left\{e_{1}, e_{2}, \ldots, e_{t}\right\}(t \geq 2), x_{i j l}^{k}$ represents the linguistic variable evaluation of alternative $a_{i}$ under criteria $u_{j}$ in state $f_{l}^{j}$. We select the optimal scheme ranking under 
a cloud prospect decision framework with a higher level of consensus degree.

2.1. Cloud Model. The cloud model reflects uncertain phenomena in the field of natural and social sciences. The usual cloud model [39] includes the complete cloud, left half cloud, and right half cloud models. It is one of the most powerful tools for characterizing the linguistic atom. The variates are assumed to be uniformly normally distributed. The complete cloud produces qualitative concepts with complete features. The derivative cloud model adds one or more parameters, generating a different form of the cloud model, such as triangular, trapezoidal, $\Gamma$, and other clouds. The cloud model is usually denoted by a cloud droplet, $C(\mathrm{Ex}, \mathrm{En}, \mathrm{He})$, where Ex is the expected value, which reflects the mathematical expectation of the qualitative concept; En is the entropy, reflecting the fuzzy qualitative concept; and He is the hyper entropy, which reflects the randomness of dispersion and the degree of certainty.

A single cloud droplet does not impact overall cloud characteristics, but the cloud droplet distribution reflects the ambiguity and randomness of cloud mapping. For effective integration of ambiguity and randomness, the universal law for most basic linguistic values in natural language can be defined as follows.

Definition 1. Suppose that $C$ is the qualitative concept of a quantitative universe $U$. For $\forall x \in U$, the membership degree, $\mu_{C}(x)$, of $C$ is a stochastic number with a stable random tendency. The membership degree $\mu_{C}(x)$ of $x$ is called the cloud on the universe $U$, denoted by $C(U)$; namely, $\mu_{C}(x)$ : $U \rightarrow[0,1], \forall x \in U$, for which $x \rightarrow \mu_{C}(x)$.

Definition 2. Let $\widetilde{A}$ be the qualitative concept in the universe $U$. A random instance $x$ in $\widetilde{A}, x \in U$, satisfies $x \sim N(\mathrm{Ex}$, $\left.\mathrm{En}^{\prime 2}\right), \mathrm{En}^{\prime} \sim N\left(\mathrm{En}, \mathrm{He}^{2}\right)$. When the certainty degree of $x$ can be expressed as $y=\exp \left[-(x-\mathrm{Ex})^{2} / 2 \mathrm{En}^{\prime 2}\right]$, the distribution of $x$ in $U$ is called the normal cloud.

Definition 3. Let $C_{1}\left(\mathrm{Ex}_{1}, \mathrm{En}_{1}, \mathrm{He}_{1}\right)$ and $C_{2}\left(\mathrm{Ex}_{2}, \mathrm{En}_{2}, \mathrm{He}_{2}\right)$ be one-dimensional normal clouds in $U$. The Hamming distance between $C_{1}$ and $C_{2}$ is defined by

$$
D\left(C_{1}, C_{2}\right)=\sqrt{\frac{\underline{d}^{2}\left(C_{1}, C_{2}\right)+\bar{d}^{2}\left(C_{1}, C_{2}\right)}{2}},
$$

where

$$
\begin{aligned}
& \underline{d}\left(C_{1}, C_{2}\right) \\
& \quad=\left|\left(\mathrm{Ex}_{1}-3 \sqrt{\mathrm{En}_{1}^{2}+\mathrm{He}_{1}^{2}}\right)-\left(\mathrm{Ex}_{2}-3 \sqrt{\mathrm{En}_{2}^{2}+\mathrm{He}_{2}^{2}}\right)\right| \\
& \bar{d}^{2}\left(C_{1}, C_{2}\right) \\
& \quad=\left|\left(\mathrm{Ex}_{1}+3 \sqrt{\mathrm{En}_{1}^{2}+\mathrm{He}_{1}^{2}}\right)-\left(\mathrm{Ex}_{2}+3 \sqrt{\mathrm{En}_{2}^{2}+\mathrm{He}_{2}^{2}}\right)\right| .
\end{aligned}
$$

In the decision process, the number of clouds has one-toone correspondence with the linguistic variable value in $U$.
Therefore, most previous studies have used the golden section method to generate clouds [36]. However, this approach has some defects, so we instead use a method that can reinforce discrimination [38].

2.2. Prospect Decision Theory Based on the Cloud Model. The cloud model combines uncertainty and ambiguity. Under the cloud model and prospect decision framework, DMs evaluate the alternatives against a reference point to estimate gain or loss and are more sensitive to loss. Prospect theory was proposed in 1979. The core concept is a prospect value, which includes a value and weight function, which reflects the bounded rationality of the DMs in the decision process. Many empirical studies have found that psychological behavior plays an important role in decision analysis. Compared with expected utility theory of uncertainty decision, prospect decision theory based on the cloud model proposed the reference point on the basis of the different effect from the reference point [39].

In prospect decision theory based on the cloud model (cloud prospect decision theory), a cloud prospect decision matrix $V_{i j}^{k}$, for alternative $i$ of attribute $j$, is constructed for every DM. This matrix is composed of a cloud prospect value function, $v(C)$, and a cloud prospect value weight, $\pi(p)$. The prospect decision matrix of every DM is

$$
V_{i j}^{k}=\sum_{l=1}^{s} \sum_{z=1, z \neq i}^{m} \pi_{i j l}^{k}\left(p_{l}^{j}\right) v\left(C_{z j l}^{k}\right)
$$

where $v(C)$ is formed by the subjective perception of the DMs [10]:

$$
v\left(C_{i j l}^{k}\right)= \begin{cases}\left(D\left(C_{i j l}^{k}, C_{z j l}^{k}\right)\right)^{\alpha}, & C_{i j l}^{k} \geq C_{z j l}^{k} \\ -\lambda\left(D\left(C_{i j l}^{k}, C_{z j l}^{k}\right)\right)^{\gamma}, & C_{i j l}^{k}<C_{z j l}^{k},\end{cases}
$$

and $\pi(p)$ reflects the probability weight function considering the DM's risk attitude:

$$
\pi_{i j}^{k}\left(p_{l}^{j}\right)= \begin{cases}\frac{\left(p_{l}^{j}\right)^{\tau}}{\left[\left(p_{l}^{j}\right)^{\tau}+\left(1-p_{l}^{j}\right)^{\tau}\right]^{1 / \tau}}, & C_{i j l}^{k} \geq C_{z j l}^{k} \\ \frac{\left(p_{l}^{j}\right)^{\delta}}{\left[\left(p_{l}^{j}\right)^{\delta}+\left(1-p_{l}^{j}\right)^{\delta}\right]^{1 / \delta}}, & C_{i j l}^{k}<C_{z j l}^{k},\end{cases}
$$

where $C_{i j l}^{k}$ denotes the cloud evaluation of alternative $i$ of attribute $j$ for the $k$ th DM. Equation (4) suggests that DM $k$, for the same attribute $j$ under the same state $l$, compares the size of the cloud droplet for alternatives $i$ and $z(z \neq$ $i)$ and calculates the distance between the cloud droplets, $D\left(C_{i j l}^{k}, C_{z j l}^{k}\right)$. The parameters $0 \leq \alpha \leq 1,0 \leq \gamma \leq 1$, which represent the degree of concavity and convexity, respectively, of the regional value function for gains and losses, control the value function, $v\left(C_{i j l}^{k}\right)$, of the DM's subjective perception. $\lambda$ is the loss aversion coefficient. When $\lambda>1$, the DM is more sensitive to loss, that is, loss averse. 


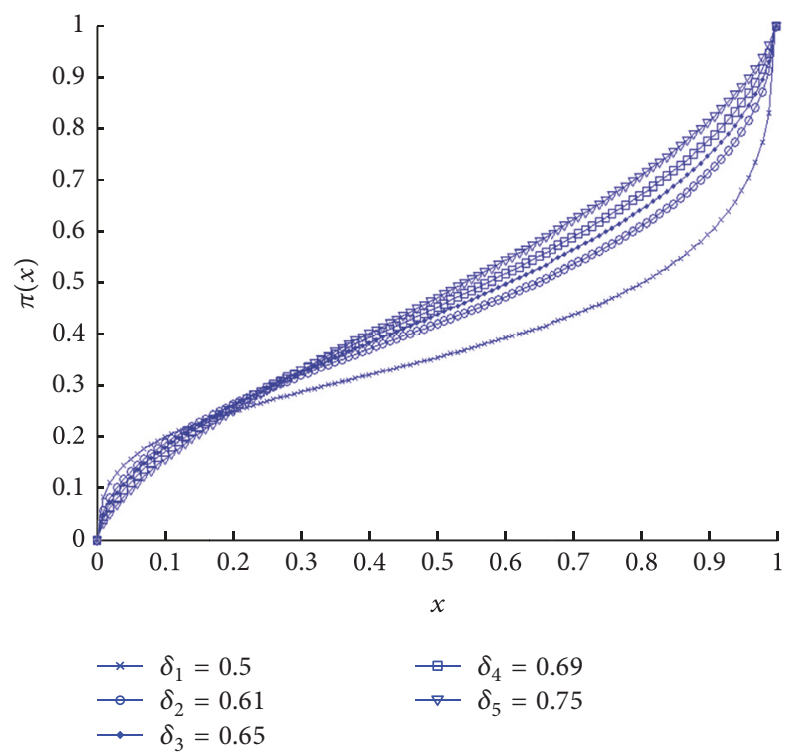

FIgURE 1: Effect of $\delta$ on the weight function $\pi(x)$.

The probability weight is the subjective judgment depending on the possibility $P$ of certain events. The risk attribute coefficients $\tau$ and $\delta$ control the curvature of the prospect weight function. $\pi(P)$ is the monotone increasing function of probability $P$. When $P$ is very small, the DM could overestimate the slight probability of a given incident. When $P$ is large, $\pi(P)<P$, illustrating that DMs overlook large probability events [39]. Equation (5) shows that, for the same attribute $j$ under the same state $l$, when the linguistic evaluation value $C_{i j l}^{k}$ of alternative $i$ is greater than that of alternative $z$, the weight function is controlled by $\tau$, which represents the risk revenue attribute coefficient. Larger $\tau$ means more adventurous DM behavior. In contrast, $\delta$ is the risk loss attribute coefficient, and Figure 1 shows the effect of $\delta$ on $\pi(x)$.

Prospect theory states that DMs measure the gain or loss according to the distance between the result and the expectation, which is sensitive to the selection of reference points. Equations (4) and (5) use dynamic reference points, which may contain both loss and income, unlike fixed reference points that include only one of these factors. An erroneous selection could lead to deviation in the overall results.

\section{Cloud Prospect Group Decision Model}

Group decisions are affected by knowledge and preferences, and decisions may have to incorporate serious differences of opinion. On the basis of the cloud model and prospect decision theory, we constructed a prospect value consistency model that includes the cloud prospect value consistency degree and feedback regulation by integrating the complete decision procedure committed to solving the group decision making for the risky multiattribute linguistic variable assessment problem. Combining the randomness and ambiguity of uncertain linguistic variable assessment with DM risk attitude and bounded rationality of psychological behavior, the proposed cloud prospect decision (CPD) making model integrates the theoretical advantages of the cloud model and prospect theory.

Aggregation, consistency measurement, and consistency model are the three essential factors of the proposed CPD model, based on the cloud prospect value method. We propose an aggregation method reflecting the concentration trend and cloud prospect consensus degree measurement. The proposed improved feedback adjustable mechanism, that is, the consistency model based on regret theory, compensates for prospect theory. The three theoretical methods together constitute the core and innovation of the proposed CPD model.

3.1. Cloud Prospect Decision (CPD) Making. The proposed $\mathrm{CPD}$ model aggregates the DMs and constructs a prospect value consensus degree through two procedures. Essentially, it aggregates DMs and attribute levels. DM aggregation provides a comprehensive decision result for each alternative $i$ and attribute $j$ for multiple DMs based on the average decision value. Based on the cloud model, CPD operates at the DM level using a set of consistency thresholds to provide a preliminary correction scheme.

(1) Aggregation. Experts from a variety of professions and preferences contribute to a decision under a complicated and uncertain environment, which is the origin of the group decision. With many correlate indicators to consider, reducing and eliminating the criteria subjective weights has a significant role in reaching a high consensus degree for the decision. Therefore, the discrepant preference information of individual assessment, $V_{i j}^{k}$, should be aggregated to a collective assessment, $V_{i j}^{c}$. erties:

The aggregation operator must satisfy the following prop-

(a) If all the assessments are the same, then the final group assessment is any one of them.

(b) The integration results are independent of the order of the experts.

(c) The group assessment opinions are the concentration trend of individual opinions.

Group assessment opinion mainly depends on the concentration trend of personal opinions. Therefore, a reasonable individual assessment integration method requires the average of multiple expert opinions. We calculate the cloud prospect decision matrix, $V_{i j}^{k}$, for each DM:

$$
V_{i j}^{c}=\frac{\sum_{k=1}^{t} V_{i j}^{k}}{t},
$$

where $t$ is the total number of the DMs. We use the cloud prospect decision matrix to construct the decision matrix for each alternative for each DM and extract the decision matrix for all the DMs according to the alternatives.

Finally, we use (6) to obtain $V_{i j}^{c}$ for each alternative $i$ and attribute $j$. 
(2) Cloud Prospect Value Consistency Measurement. Classically, consensus is defined as the full and unanimous agreement of all decision makers regarding all possible alternatives [40]. However, complete agreement is not always necessary in real situations. Therefore, to obtain the maximum consensus degree among DMs on the solution set of alternatives [41], we build a CPD model:

$$
\begin{aligned}
& \operatorname{CPD}\left(V_{i}^{k}\right) \\
& =\sqrt{\frac{1}{n} \sum_{j=1}^{n}\left(\frac{V_{i j}^{k}-V_{i j}^{c}}{\left\{\left[\max _{g}\left(V_{i g}^{k}-V_{i g}^{c}\right)-\min _{g}\left(V_{i g}^{k}-V_{i g}^{c}\right)\right]+\varepsilon\right\} * n}\right)^{2}},
\end{aligned}
$$

where $\varepsilon$ is a correction coefficient, usually assigned a value of 1 ; and $g=1,2, \ldots, n$. The CPD of alternative $i$ for all DMs is

$$
\operatorname{CPD}\left(a_{i}\right)=1-\frac{1}{t} \sum_{k=1}^{t} \operatorname{CPD}\left(V_{i}^{k}\right)
$$

Let the consensus degree threshold of CPD for group decisions be $\beta$. If $\operatorname{CPD}\left(a_{i}\right)<\beta$, then adjust $a_{i}$ to minimize CPD and adjust the alternatives.

3.2. Feedback Regulation Mechanism. When the CPD does not satisfy the acceptable threshold $\beta$, the feedback regulation aims to provide corresponding adjustment suggestions to guide DM's correction. This feedback adjustment is intended to improve the efficiency of sorting decision schemes. The targeted adjustment alternative is selected by the regretrejoice model [8]. Prospect theory makes full use of the bounded rationality and risk aversion of decision makers. Contrasting with the collective decision, there will be disparity between different assessments which will cause regret (loss) or rejoice (gain). Therefore, we take advantage of the regret concept for feedback and adjust the scheme with the known inconsistency information. Regret theory [42, 43] assumes that the DMs pay attention not only to results obtained by choosing alternatives but also to the outcome of other alternatives and avoid choosing alternatives that will make them regret.

Regret theory has been successfully applied for investment choice [44, 45] and decision making [42] and has shown that anticipated disutility from regret can have a potent effect on DMs. Thus, regret increases the regret averse attitude of an expert's willingness to adjust the scheme's assessment after knowing the difference between individual evaluation and collective evaluation until the optimal alternative ranking is finally achieved. The feedback adjustment information enables reevaluation at different state levels and includes four steps.

(1) Calculate Regret-Rejoice Value. Using $V_{i j}^{k}$, calculate the disparity between the individual and collective decision matrixes. The regret-rejoice value is

$$
\begin{gathered}
R(\Delta V)=1-e^{-\xi \Delta V}, \\
\Delta V=V_{i j}^{k}-V_{i j}^{c},
\end{gathered}
$$

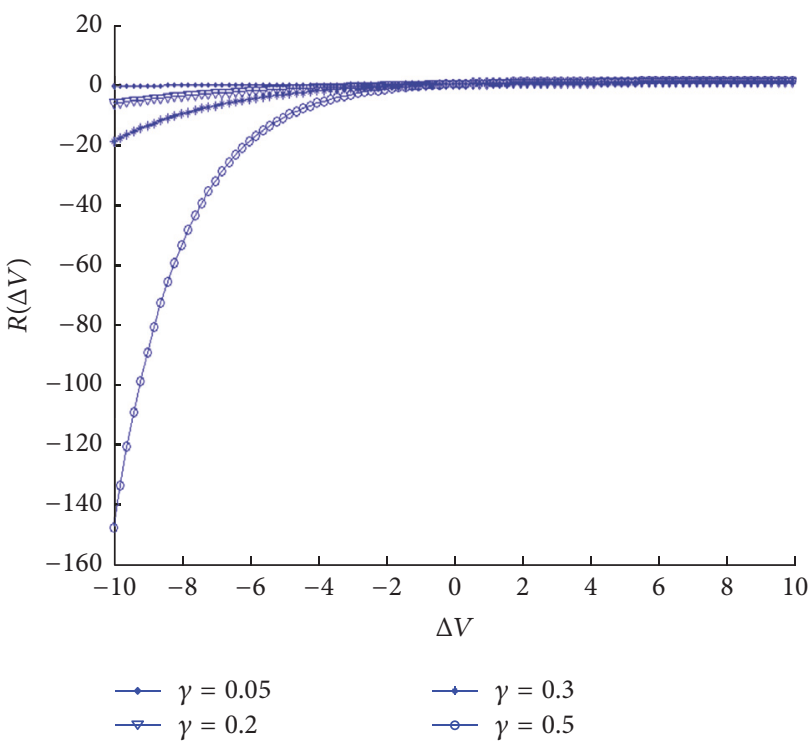

FIGURE 2: Effect of $\gamma$ on the regret-rejoice value.

where $\xi$ is the DM risk aversion coefficient. Larger $\xi$ implies greater degree of DM risk aversion. Equation (10) denotes the difference between the prospect value of alternative $a_{i}$ and attribute $u_{j}$ for DM $k$. When $R(\Delta V)>0, R(\Delta V)$ denotes rejoice, and when $R(\Delta V)<0, R(\Delta V)$ denotes regret [19]. Similar to prospect theory, the DM is more sensitive to negative than to positive prospect, derived from psychological perception. Figure 2 shows the effect of $\xi$ on $R(\Delta V)$.

(2) Lock the Feedback Scheme. Choose the minimum regretrejoice value as the amended attribute of the adjustment alternative:

$$
\min R(\Delta V)= \begin{cases}\min R(\Delta V) & R(\Delta V)<0 \\ \text { or } & \\ \min R(\Delta V) & R(\Delta V)>0 .\end{cases}
$$

Assuming $V_{i j}^{c}$ to be the authoritative benchmark of the prospect value, when $\Delta V_{0}<0$, smaller values imply larger disparity between the individual and group prospect value matrixes. Smaller $R(\Delta V)$ implies more regret the DM feels for the evaluation, and larger $R(\Delta V)$ implies more desirable individual adjustment of the evaluation value.

Similarly, when $R(\Delta V) \geq 0$, larger values imply more satisfaction with the individual decision, and the DM often rejoices over underestimating the value of the alternative on the surface. However, they have known the defect of the alternative, so, the less desirable adjust the prospect value decision matrix of the alternative. Thus, they will keep the original assessment.

(3) Calculate Guidance Information for Feedback Adjustment. Compute the cloud prospect values from (3)-(5). When the feedback correction of prospect value matrix $V_{i j}^{k}$ is locked, the target correction matrix is determined. If the prospect 


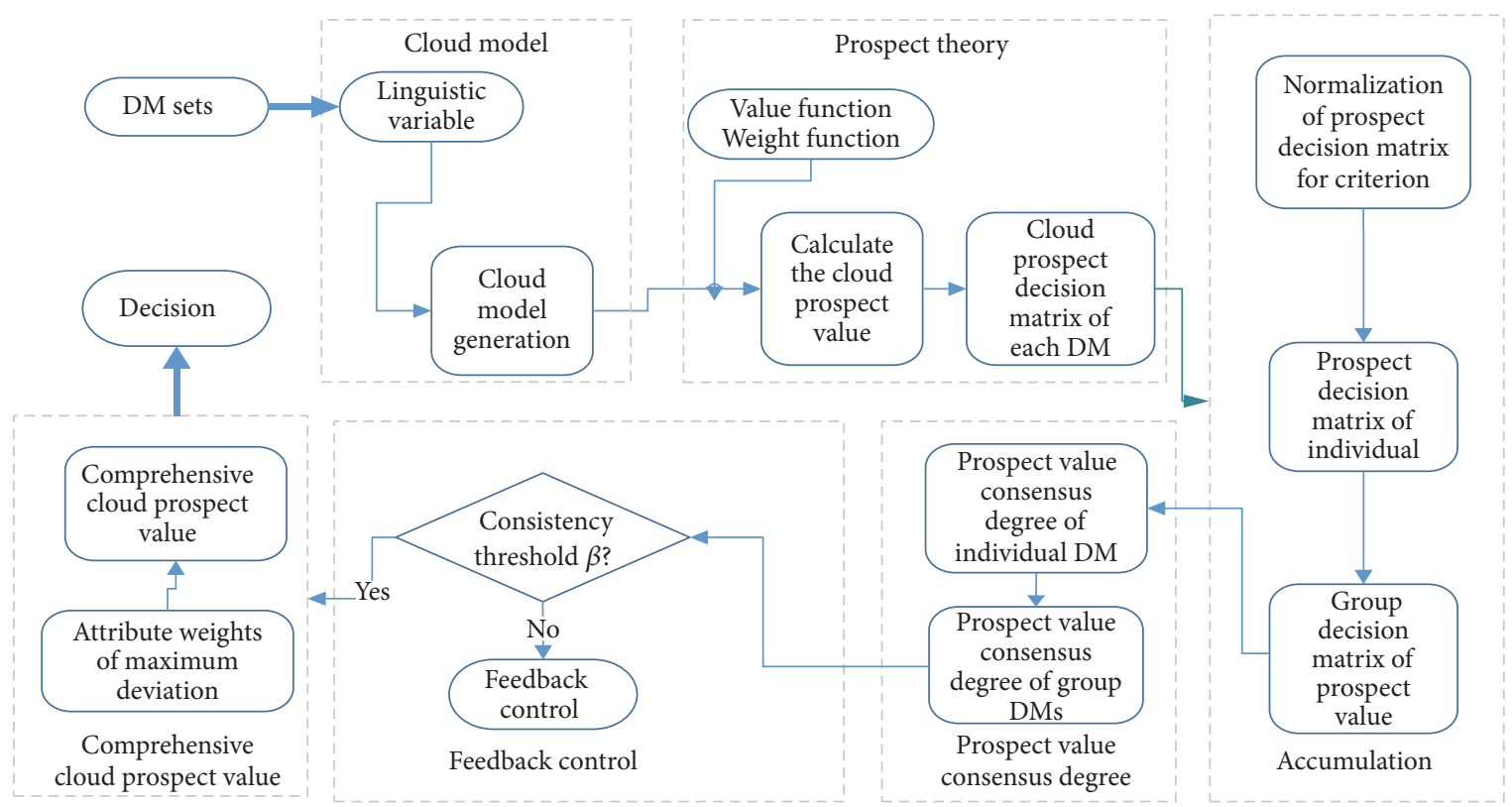

FIGURE 3: Group decision structure under the proposed cloud prospect and regret feedback procedure.

value $V_{i j}^{k}>V_{i j}^{c}$, then DM $k$ should reduce $x_{i j l}^{k}$. Otherwise, the equality in (13) indicates that the DM could refuse to correct $x_{i j l}^{k}$.

$$
\text { If } V_{i j}^{k}<V_{i j}^{c} \text {, then DM } k \text { should increase } x_{i j l}^{k} \text { : }
$$

$$
\begin{aligned}
& \text { if } V_{i j}^{k}>V_{i j}^{c}, \text { then }\left(x_{i j l}^{k}\right)^{\prime} \leq x_{i j l}^{k} ; \\
& \text { if } V_{i j}^{k}=V_{i j}^{c}, \text { then }\left(x_{i j l}^{k}\right)^{\prime}=x_{i j l}^{k} ; \\
& \text { if } V_{i j}^{k}<V_{i j}^{c}, \text { then }\left(x_{i j l}^{k}\right)^{\prime} \geq x_{i j l}^{k},
\end{aligned}
$$

where $\left(x_{i j l}^{k}\right)^{\prime}$ is the linguistic evaluation value after adjustment.

CPD provides a complete feedback regulation mechanism. It determines the consensus degree of the group decision at the alternative level and determines the program to be modified by comparing the CPD of all alternatives. CPD subdivides alternatives into attributes and confirms the attribute with the minimum regret-rejoice value. The feedback regulation of (12)-(14) ensures that the DMs are directed to reevaluate the alternatives and repeat all the procedures until all the alternatives satisfy $\beta$. The DMs can also refuse to reevaluate their decisions. Figure 3 shows the CPD model; Figure 4 shows the DM feedback regulation.

3.3. Decision Procedure. Under the multicriteria group decision environment, the group decision procedure based on prospect theory and the cloud model is as follows:

(1) The linguistic variables are transformed into a normal cloud model: following the DM linguistic evaluation criteria, the corresponding cloud model is combined with the effective universe, $U=\left[X_{\min }, X_{\max }\right]$, as discussed in Section 3.1.
(2) A cloud prospect decision matrix is constructed for each alternative: the cloud prospect decision matrix, $\left(V_{i j}^{k}\right)_{m \times n}$, is calculated for each DM for each alternative and attribute following (1)-(5).

(3) The cloud prospect decision matrixes are aggregated: we calculate the cloud prospect decision matrix of the group based on each DM's cloud prospect decision matrix.

(4) Compute the CPD: the consensus degree, $\operatorname{CPD}\left(e_{k}\right)$, is calculated for each DM. For a cloud prospect consensus degree threshold $\beta$, when $\mathrm{CPD} \geq \beta$ for all alternatives or the DM refuses to correct $x_{i j l}^{k}$, proceed to Step (6); otherwise, proceed to Step (5).

(5) Control feedback: calculate the regret or rejoice value for all the alternatives for different criteria following (9) and (10). Lock feedback for the criteria with the minimum regret-rejoice value using (11) and provide correction and instruction information to the DMs using (12)-(14).

(6) Calculate weights to maximize deviation: the difference between individual and collective experts affects criteria weights. Maximizing deviation makes use of individual and group outputs to maximize this difference. Some criteria play an important role in the sorting of the schemes and should be endowed a larger weight coefficient. Therefore, in addition to the basic constraints, $\sum_{j=1}^{n} \omega_{j}=1$, we add reasonable constraints to obtain optimal weight coefficients $\omega^{*}=$ $\left(\omega_{1}^{*}, \omega_{2}^{*}, \ldots, \omega_{n}^{*}\right)$ :

$$
\max W=\sum_{i=1}^{m-1} \sum_{k=i+1}^{m} \sum_{j=1}^{n}\left|V_{i j}^{c}-V_{k j}^{c}\right| \cdot \omega_{j} .
$$




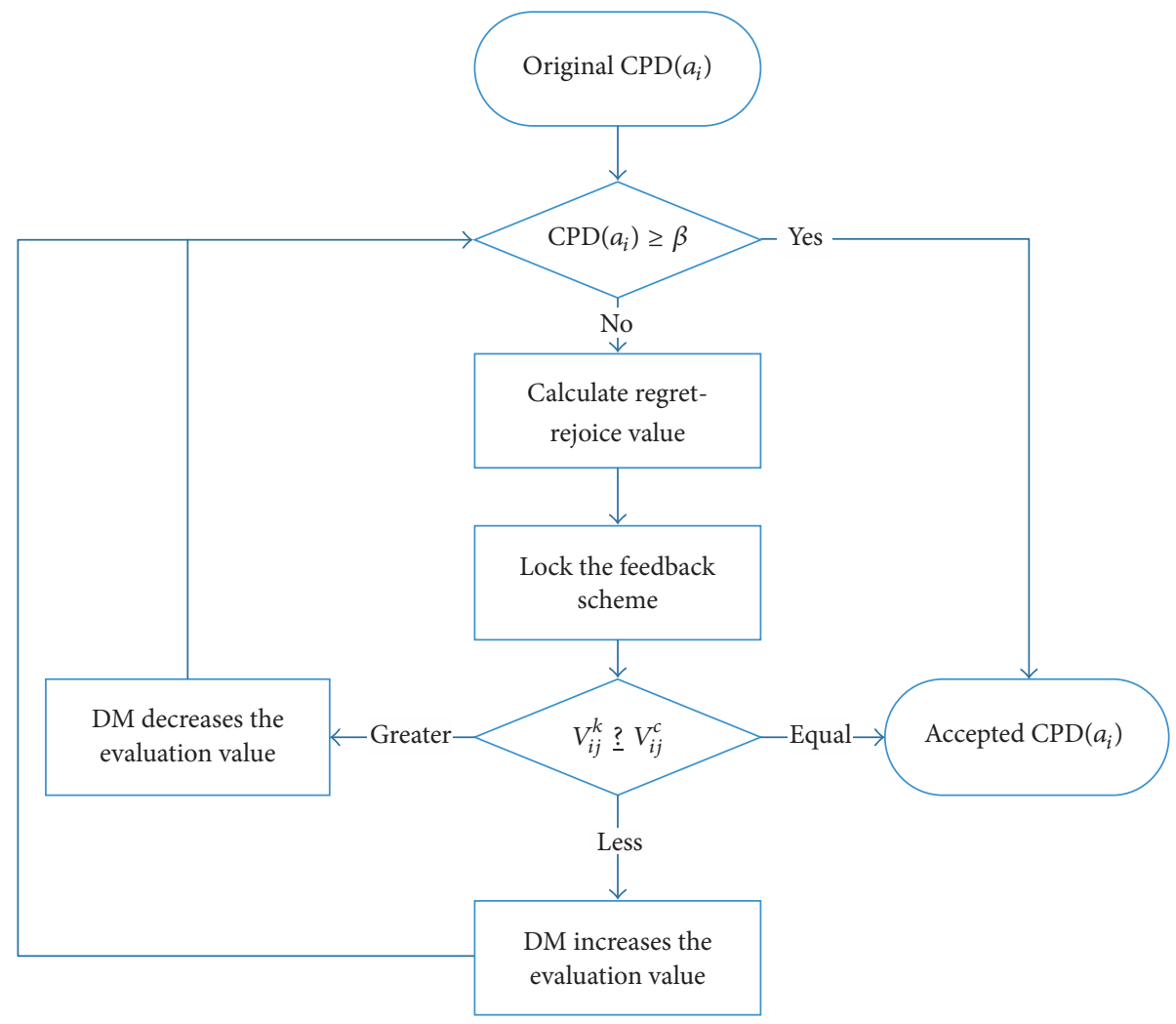

FIGURE 4: Feedback control diagram of DMs.

TABLE 1: Linguistic prospect decision matrix of $e_{1}{ }^{*}$.

\begin{tabular}{ccccccccccccccccccccc}
\hline & & & $q_{1}$ & & & \multicolumn{1}{c}{$q_{2}$} & & & \multicolumn{1}{c}{$q_{3}$} & & $q_{4}$ \\
& $f_{1}^{1}$ & $f_{2}^{1}$ & $f_{3}^{1}$ & $f_{4}^{1}$ & $f_{5}^{1}$ & $f_{1}^{2}$ & $f_{2}^{2}$ & $f_{3}^{2}$ & $f_{4}^{2}$ & $f_{5}^{2}$ & $f_{1}^{3}$ & $f_{2}^{3}$ & $f_{3}^{3}$ & $f_{4}^{3}$ & $f_{5}^{3}$ & $f_{1}^{4}$ & $f_{2}^{4}$ & $f_{3}^{4}$ & $f_{4}^{4}$ & $f_{5}^{4}$ \\
\hline$a_{1}$ & 2 & 2 & 2 & 3 & 3 & 2 & 1 & 3 & 2 & 2 & 3 & 5 & 1 & 3 & 4 & 1 & 2 & 4 & 3 & 5 \\
$a_{2}$ & 2 & 3 & 1 & 5 & 2 & 1 & 3 & 1 & 4 & 2 & 2 & 3 & 3 & 5 & 3 & 2 & 2 & 3 & 2 & 3 \\
$a_{3}$ & 3 & 1 & 3 & 2 & 4 & 2 & 1 & 4 & 2 & 4 & 2 & 4 & 1 & 5 & 4 & 2 & 1 & 3 & 3 & 5 \\
$p$ & 0.1 & 0.2 & 0.5 & 0.1 & 0.1 & 0.05 & 0.1 & 0.6 & 0.15 & 0.1 & 0.07 & 0.25 & 0.35 & 0.2 & 0.13 & 0.15 & 0.15 & 0.3 & 0.2 & 0.2 \\
\hline
\end{tabular}

${ }^{*} e$ represents the first DM; $f$ means the natural state; $p$ represents the occurrence probability of the attribute; $q_{1}-q_{4}$ represent the four attributes of alternatives; $a_{1}-a_{3}$ mean the three alternatives.

(7) Compute the comprehensive prospect value: given the attribute weights $\omega_{j}, j=1,2, \ldots, n$, for the DMs, we compute the comprehensive prospect value of each based on the comprehensive prospect value. The optimal alternative with the greatest comprehensive prospect value is

$$
V_{i}=\sum_{j=1}^{n} \omega_{j} V_{i j}^{c}, \quad i=1,2, \ldots, m .
$$

\section{Numerical Analysis and Discussion}

Investment in shipping vessels is one of the most important business activities in international shipping and involves large amounts of money, a long investment recovery period, and a significant risk level.

Consider a ship owner who intends to invest in one of three dry cargo ships: $a_{1}$ is a bulk carrier, $a_{2}$ is a container ship, and $a_{3}$ is a multipurpose ship. DMs evaluate the direct $(u 1)$, indirect $(u 2)$, social benefit $(u 3)$, and pollution loss $(u 4)$. Each of these four criteria is divided into five possible natural states: $f_{1}^{j}=$ very good, $f_{2}^{j}=$ good, $f_{3}^{j}=$ fair, $f_{4}^{j}=$ poor, and $f_{5}^{j}=$ very poor. Then attribute $Q$ has occurrence probability $P$ under natural state $f$, and the linguistic evaluation set $S=\{1,2,3,4,5\}$ represents $\{$ very poor, poor, fair, good, very good\}, respectively. Three experts from marketing, finance, and industry make independent linguistic evaluations, as shown in Tables 1-3. 
TABLE 2: Linguistic prospect decision matrix of $e_{2}$.

\begin{tabular}{ccccccccccccccccccccc}
\hline & & & $q_{1}$ & & & & & $q_{2}$ & & & & $q_{3}$ & & & & $q_{4}$ \\
& $f_{1}^{1}$ & $f_{2}^{1}$ & $f_{3}^{1}$ & $f_{4}^{1}$ & $f_{5}^{1}$ & $f_{1}^{2}$ & $f_{2}^{2}$ & $f_{3}^{2}$ & $f_{4}^{2}$ & $f_{5}^{2}$ & $f_{1}^{3}$ & $f_{2}^{3}$ & $f_{3}^{3}$ & $f_{4}^{3}$ & $f_{5}^{3}$ & $f_{1}^{4}$ & $f_{2}^{4}$ & $f_{3}^{4}$ & $f_{4}^{4}$ & $f_{5}^{4}$ \\
\hline$a_{1}$ & 3 & 4 & 2 & 1 & 3 & 4 & 2 & 5 & 2 & 1 & 3 & 4 & 2 & 1 & 3 & 2 & 3 & 2 & 1 & 4 \\
$a_{2}$ & 2 & 4 & 1 & 5 & 2 & 1 & 3 & 2 & 1 & 4 & 2 & 5 & 3 & 1 & 2 & 4 & 3 & 2 & 1 & 4 \\
$a_{3}$ & 4 & 2 & 1 & 5 & 3 & 2 & 1 & 4 & 2 & 1 & 5 & 3 & 2 & 1 & 4 & 2 & 1 & 2 & 3 & 5 \\
$p$ & 0.2 & 0.2 & 0.3 & 0.2 & 0.1 & 0.1 & 0.1 & 0.5 & 0.2 & 0.1 & 0.1 & 0.3 & 0.35 & 0.1 & 0.15 & 0.1 & 0.1 & 0.5 & 0.2 & 0.1 \\
\hline
\end{tabular}

TABLE 3: Linguistic prospect decision matrix of $e_{3}$.

\begin{tabular}{ccccccccccccccccccccc}
\hline & & & $q_{1}$ & & & \multicolumn{1}{c}{$q_{2}$} & & & \multicolumn{1}{c}{$q_{3}$} & & $q_{4}$ \\
& $f_{1}^{1}$ & $f_{2}^{1}$ & $f_{3}^{1}$ & $f_{4}^{1}$ & $f_{5}^{1}$ & $f_{1}^{2}$ & $f_{2}^{2}$ & $f_{3}^{2}$ & $f_{4}^{2}$ & $f_{5}^{2}$ & $f_{1}^{3}$ & $f_{2}^{3}$ & $f_{3}^{3}$ & $f_{4}^{3}$ & $f_{5}^{3}$ & $f_{1}^{4}$ & $f_{2}^{4}$ & $f_{3}^{4}$ & $f_{4}^{4}$ & $f_{5}^{4}$ \\
\hline$a_{1}$ & 2 & 4 & 3 & 1 & 5 & 2 & 3 & 4 & 1 & 2 & 5 & 3 & 2 & 1 & 4 & 2 & 5 & 1 & 3 & 4 \\
$a_{2}$ & 4 & 2 & 1 & 5 & 3 & 2 & 5 & 4 & 1 & 2 & 3 & 5 & 4 & 1 & 2 & 1 & 2 & 3 & 5 & 4 \\
$a_{3}$ & 4 & 1 & 3 & 2 & 5 & 2 & 2 & 2 & 1 & 4 & 2 & 3 & 5 & 4 & 1 & 4 & 3 & 5 & 1 & 2 \\
$p$ & 0.1 & 0.3 & 0.2 & 0.2 & 0.2 & 0.2 & 0.3 & 0.1 & 0.2 & 0.2 & 0.2 & 0.2 & 0.3 & 0.1 & 0.2 & 0.2 & 0.2 & 0.4 & 0.1 & 0.1 \\
\hline
\end{tabular}

TABLE 4: Numerical characteristics of cloud model.

\begin{tabular}{lccc}
\hline Cloud model & $E x$ & $E n$ & $H e$ \\
\hline$C_{+2}\left(E x_{+2}, E n_{+2}, H e_{+2}\right)$ & 10 & 2.0604 & 0.1309 \\
$C_{+1}\left(E x_{+1}, E n_{+1}, H e_{+1}\right)$ & 3.82 & 1.2733 & 0.0809 \\
$C_{0}\left(E x_{0}, E n_{0}, H e_{0}\right)$ & 0 & 0.7869 & 0.05 \\
$C_{-1}\left(E x_{-1}, E n_{-1}, H e_{-1}\right)$ & -3.82 & 1.2733 & 0.0809 \\
$C_{-2}\left(E x_{-2}, E n_{-2}, H e_{-2}\right)$ & -10 & 2.0604 & 0.1309 \\
\hline
\end{tabular}

The optimal dry cargo ship investment decision is made following the proposed CPD procedures described above:

(a) Linguistic variable transformation: the linguistic evaluation set is transformed to five cloud models on the universe $U=[-10,10]$, that is, $\left\{C_{+2}, C_{+1}, C_{0}\right.$, $\left.C_{-1}, C_{-2}\right\}$. The numerical characteristics of the five clouds are shown in Table 4

(b) Producing the cloud prospect decision matrix: the cloud prospect decision matrix of individual $V^{k}$ and group $V^{c}$ is created following Steps (2) and (3). To obtain better distinction, we set $\alpha=\gamma=0.88, \lambda=$ $2.25, \tau=0.61$, and $\delta=0.69$, (in the following matrixes, 0.0000 denotes a very small value, not necessarily 0 ):

$$
\begin{aligned}
V^{1} & =\left(\begin{array}{llll}
0.7403 & 0.0142 & 0.1547 & 0.0000 \\
1.0000 & 1.0000 & 0.0002 & 1.0000 \\
0.0017 & 0.0000 & 1.0000 & 0.0005
\end{array}\right) \\
V^{2} & =\left(\begin{array}{llll}
0.7647 & 0.0000 & 1.0000 & 1.0000 \\
1.0000 & 1.0000 & 0.0000 & 0.0034 \\
0.0193 & 0.0250 & 0.0018 & 0.0000
\end{array}\right),
\end{aligned}
$$

$$
\begin{aligned}
V^{3} & =\left(\begin{array}{llll}
0.0010 & 0.6907 & 0.2891 & 0.5786 \\
0.0617 & 0.0000 & 0.1350 & 1.0000 \\
1.0000 & 1.0000 & 1.0000 & 0.0000
\end{array}\right), \\
V^{C} & =\left(\begin{array}{rrrr}
-8.7761 & -10.1200 & -5.5585 & -6.1984 \\
-7.1995 & -4.9391 & -11.5442 & -3.2871 \\
-9.7045 & -7.5947 & -6.6376 & -12.4365
\end{array}\right) .
\end{aligned}
$$

(c) Aggregation and consistency measurement: individual and group CPDs are calculated based on a consensus degree threshold of $\beta=0.9$ following Step (4), using (7) and (8). The original CPD of each alternative is listed in the second column of Figure 5, where the cylinders denote that the consistency degree threshold was satisfied, that is, passes consistency; and dashed rectangles represent a consistency degree below the lower limit, which do not pass the check. Although consistency does not meet the threshold in the solid rectangles, this iteration is temporarily permitted. To achieve a higher consistency threshold, all the alternatives iteratively proceed to feedback adjustment until reaching 0.98 threshold. The blue background graphic denotes the feedback adjusted attribute

(d) Feedback adjustment: following the feedback mechanism of Step (5), we compute the original CPD of each alternative, and $a_{1}$ and $a_{2}$ both do not reach the consistent degree threshold, 0.9. Since $a_{1}$ has the smallest CPD, we lock scheme $a_{1}$ as the correction alternative and then apply feedback control to satisfy the consistency degree. The specific feedback corrections are shown in Figure 5 


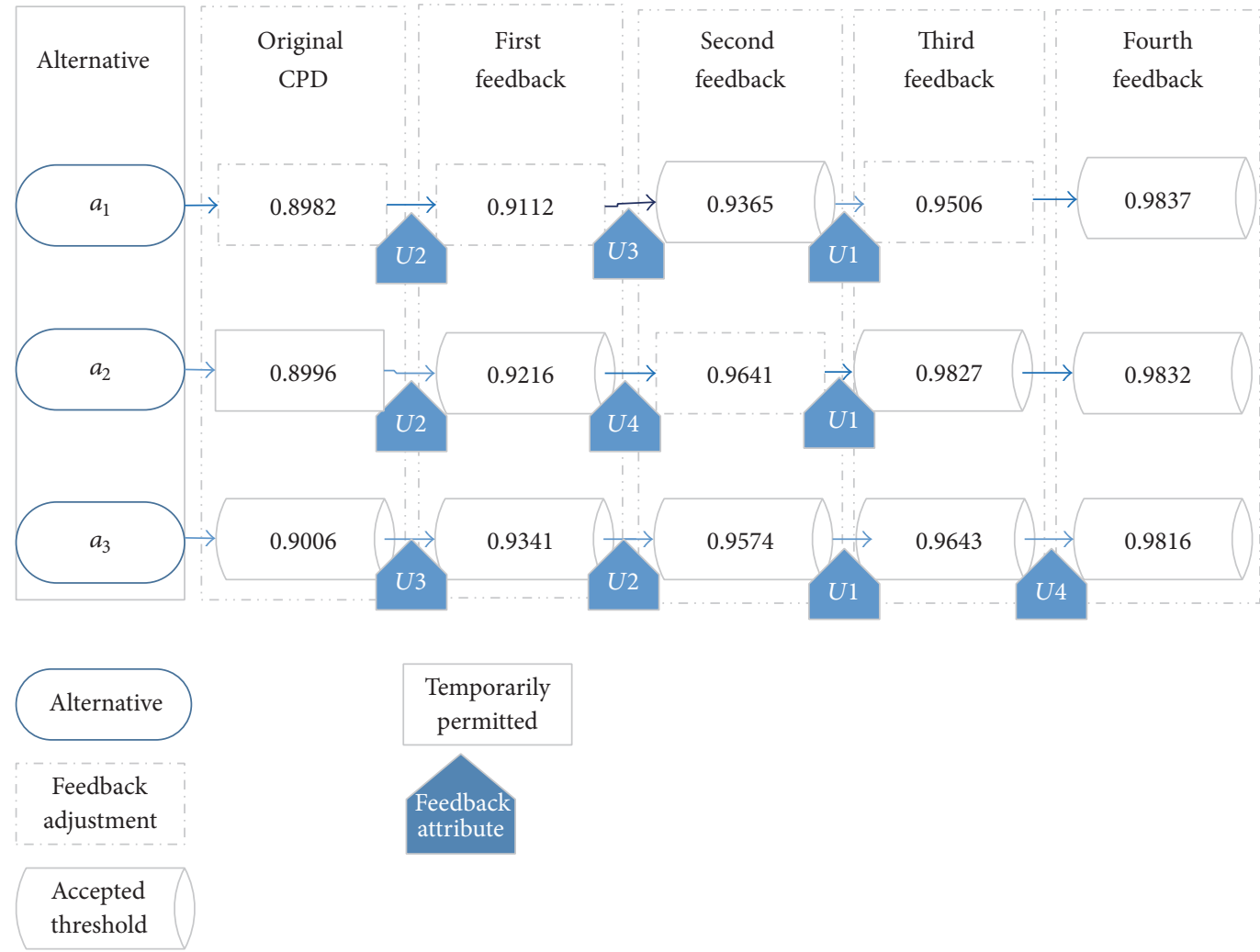

FIGURE 5: Feedback correction details.

(e) Feedback for alternative $a_{1}$ : the feedback regulation mechanism based on regret theory includes the following:

(i) Ualculate $\Delta V$, the difference between the individual and collection prospect value.

(ii) Use (10) to provide the psychological gap of regret or rejoice.

(iii) Calculate the regret-rejoice matrix, $R(\Delta V)$, from (9).

(iv) To better distinguish the effects, we set $\gamma=0.5$.

Thus,

$$
\begin{aligned}
& \Delta V_{1}=\left(\begin{array}{cccc}
2.8345 & 4.8636 & 0.0542 & -5.7282 \\
2.4006 & -13.8886 & 5.1601 & 7.3129 \\
-5.2351 & 9.0249 & -5.2142 & -1.5846
\end{array}\right) \\
& R\left(\Delta V_{1}\right) \\
& =\left(\begin{array}{cccc}
0.7576 & 0.9121 & 0.0267 & -16.5337 \\
0.6989 & -1036.2413 & 0.9242 & 0.9742 \\
-12.7022 & 0.9890 & -12.5596 & -1.2085
\end{array}\right) .
\end{aligned}
$$

(f) Lock the adjustment decision maker: from (11), decision maker $e_{2}$ has the minimum regret-rejoice value to attribute $q_{2}(-1036.2413)$, which has the greatest disparity relative to the collective DMs and indicates that $\mathrm{DM} e_{1}$ feels the maximum regret for attribute $q_{2}$. Then lock $R_{2,2}$ as the adjustment element. Provide guide information for the feedback adjustment as $V_{i j}^{k}(-24.9475)<V_{i j}^{c}(-11.0589)$ and then $\left(x_{i j l}^{k}\right)^{\prime} \geq x_{i j l}^{k}$; then increase $x_{2,2,3}$ from 3 to 4 under that state.

After the first correction, the individual and collective decision matrixes are

$$
\begin{aligned}
& \left(V^{1}\right)^{\prime} \\
& =\left(\begin{array}{cccc}
-10.9416 & 0.8795 & -9.5043 & -16.6805 \\
-5.6409 & -14.2913 & -12.3644 & -6.8879 \\
-11.9936 & -3.0713 & -8.6384 & -16.4293
\end{array}\right) \\
& \left(V^{2}\right)^{\prime} \\
& \quad=\left(\begin{array}{cccc}
-12.0808 & -5.9475 & -4.3984 & 1.1145 \\
-14.0602 & -6.8756 & -10.7037 & -6.5837 \\
-10.0540 & 1.4445 & -12.3211 & -22.2397
\end{array}\right), \\
& \left(V^{3}\right)^{\prime} \\
& \quad=\left(\begin{array}{cccc}
-19.0112 & -1.0340 & -18.4154 & -7.7830 \\
-9.8505 & -16.5835 & -11.5341 & -8.2358 \\
0.8503 & -0.8133 & -7.6361 & -28.3345
\end{array}\right),
\end{aligned}
$$


TABLE 5: Consensus for the proposed regret-rejoice feedback.

\begin{tabular}{lcccc}
\hline Consistency threshold & Round & Consistency & Feedback loops & Stop iteration \\
\hline$\beta=0.9$ & 1 & 0.8982 & 0 & No \\
$\beta=0.9$ & 2 & 0.9112 & 2 & Yes \\
$\beta=0.95$ & 3 & 0.9365 & 3 & No \\
$\beta=0.95$ & 4 & 0.9506 & 4 & Yes \\
$\beta=0.98$ & 5 & 0.9837 & 4 & Yes \\
\hline
\end{tabular}

TABLE 6: Consensus for direct feedback.

\begin{tabular}{lcccc}
\hline Consistency threshold & Round & Consistency & Feedback loops & Stop iteration \\
\hline$\beta=0.9$ & 1 & 0.8982 & 0 & No \\
$\beta=0.9$ & 2 & 0.8991 & 2 & No \\
$\beta=0.9$ & 3 & 0.9040 & 3 & Yes \\
$\beta=0.95$ & 4 & 0.9054 & 4 & No \\
$\beta=0.95$ & 5 & 0.9257 & 5 & No \\
$\beta=0.95$ & 6 & 0.9321 & 6 & No \\
$\beta=0.95$ & 7 & 0.9435 & 7 & Yes \\
$\beta=0.95$ & 8 & 0.9508 & 8 & No \\
$\beta=0.98$ & 9 & 0.9425 & 9 & No \\
$\beta=0.98$ & 10 & 0.9739 & 10 & No \\
$\beta=0.98$ & 11 & 0.9563 & & \\
\hline
\end{tabular}

$\left(V^{c}\right)^{\prime}$

$$
=\left(\begin{array}{cccc}
-14.0112 & -2.0340 & -10.7727 & -7.7830 \\
-9.8505 & -12.5835 & -11.5341 & -7.2358 \\
-7.0658 & -0.8133 & -9.5318 & -22.3345
\end{array}\right) \text {. }
$$

(g) First round of feedback adjustment results: after adjusting alternative $a_{1}, \operatorname{CPD}\left(a_{1}\right)=0.9112$ and, after the first feedback adjustment, alternatives $a_{2}$ and $a_{3}$ both satisfy the threshold $\beta=0.9$. The CPDs of all the three alternatives are improved significantly: $\operatorname{CPD}\left(a_{2}\right)=0.9216$ and $\operatorname{CPD}\left(a_{3}\right)=0.9341$. Thus, the first feedback causes the consistency degree of alternatives $a_{1}, a_{2}$, and $a_{3}$ to increase by $1.45 \%, 2.45 \%$, and $3.42 \%$, respectively

(h) High degree of consensus: to improve degree of consensus, we increased the consensus threshold to $\beta=0.95$, requiring a further feedback. Comparing the three alternative CPDs, $a_{1}$ was further locked, and after the second feedback all the schemes except $a_{2}$ satisfied the higher threshold. Assuming that the consistency was 0.98 , we repeated the procedure in procedure (e), and the iteration stopped. Figure 5 shows the detailed feedback and consistency degrees

(i) Calculating criteria weights: from Step (6), the model is $\max Z$

$$
\begin{aligned}
= & 2.1392 \omega_{1}+1.4769 \omega_{2}+1.7881 \omega_{3} \\
& +2.0539 \omega_{4}
\end{aligned}
$$

$$
\begin{array}{ll}
\text { s.t. } & \sum_{j=1}^{4} \omega_{j}=1 \\
& 0.15 \leq \omega_{1} \leq 0.2 \\
& 0.3 \leq \omega_{2} \leq 0.5 \\
& \omega_{1} \leq \omega_{4} \leq \omega_{2} \\
& 0<\omega_{3} \leq \omega_{4},
\end{array}
$$

which provides attribute weights $\omega=[0.2,0.3,0.2001$, $0.2999]$, and, from $(16), V=(-7.8111,-7.7813$, $-7.6412)$

Thus, following the maximizing principle, the optimal alternative is $a_{3}$ (multipurpose ship) and then $a_{2}$ (container ship), and the worst prospect value is $a_{1}$ (bulk carrier).

The proposed CPD method uses feedback adjustment, whereby the DMs are presented with specific feedback. The high efficiency of the proposed method is evident by comparison with direct feedback, which is the comparison of individual and group prospect decision matrices (see (12)-(14)). Consider alternative $a_{1}$ as an example, where original $C P D=0.8982$. Table 5 shows the variation of an increasing consistency degree with regret-rejoice feedback, and Table 6 shows the case for direct feedback. Direct feedback requires twice the feedback to achieve the given thresholds $(\beta=$ 0.9 ); that is, the improvement of consensus degree increases slowly. Direct feedback, after 8 feedback loops, obtains a high consensus degree, $\beta=0.95$. However, using regret theory of feedback thought, after three loops of correction, CPD has 
a higher consensus degree of 0.9506 , which satisfies a higher threshold beforehand. Again, compared to the proposed CPD method, if $\beta=0.98$, the regret feedback adjustment reaches the threshold after 4 feedback loops, whereas the direct feedback has still not reached the threshold after 10 feedback loops. Thus, the proposed CPD method shows high efficiency for the regret feedback mechanism

Consistency after regret-rejoice feedback is always improved, but consistency after direct feedback does not always increase; for example, the eighth feedback decreases. This unstable trend reduces efficiency and greatly increases the complexity of group decision making.

Thus, the proposed regret feedback method significantly reduces the number of corrections which reduces time costs and increases decision making efficiency.

\section{Conclusion}

We proposed a new method to address the problem of risky multiattribute group decisions, where group decision making is under a complicated and ambiguous environment. The decision attribute varies within an uncertain environment that changes with the situation. We propose formulating the group decision making process as a fuzzy linguistic variable evaluation and constructed a cloud prospect aggregation method, consistency measurement, and feedback mechanism for the consistency model. The proposed CPD model incorporates the advantages of the cloud model randomness and vagueness, prospect theory sensitivity to loss, and risk aversion based on a regret feedback mechanism to provide an organic cloud prospect consistency degree method. This fusion approach not only ensures group decision consistency but also guides the DMs to correct the decision matrix through an efficient feedback adjustment mechanism. The proposed method was verified by an algorithm example, showing the efficiency of feedback loops, consistency, and so forth.

\section{Conflicts of Interest}

The authors declare that they have no conflicts of interest.

\section{Acknowledgments}

This work was supported by the National Natural Science Foundation of China (51409065 and 71101034), the project of Provincial Natural Science Foundation (JJ2016QN0048), the project of Provincial Postdoctoral Fund (LBH-Z15047), and the project of the Provincial Youth Science Foundation (JJ2016QN0645).

\section{References}

[1] R. E. Steuer and P. Na, "Multiple criteria decision making combined with finance: a categorized bibliographic study," European Journal of Operational Research, vol. 150, no. 3, pp. 496-515, 2003.

[2] J. Wallenius, J. S. Dyer, P. C. Fishburn, R. E. Steuer, S. Zionts, and K. Deb, "Multiple criteria decision making, multi-attribute utility theory: recent accomplishments and what lies ahead," Management Science, vol. 54, no. 7, pp. 1336-1349, 2008.

[3] P. Liu and W. Li, "Risky multiobjective decision making theory and its application," Chinese Journal of Management Science, vol. 11, no. 6, article 1014, 2003.

[4] A. Eskandari, P. Ffolliott, and F. Szidarovszky, "Uncertainty and method choice in discrete multiobjective programming problems," Applied Mathematics and Computation, vol. 69, no. 2-3, pp. 335-351, 1995.

[5] F. H. Knight, Risk, Uncertainty and Profit, Houghton Mifflin, New York, NY, USA, 1921.

[6] J. S. H. Kornbluth, "Dynamic multi-criteria decision making," Journal of Multi-Criteria Decision Analysis, vol. 1, no. 2, pp. 8192, 1992.

[7] F. Meng, C. Tan, and X. Chen, "An approach to Atanassov's interval-valued intuitionistic fuzzy multi-attribute decision making based on prospect theory," International Journal of Computational Intelligence Systems, vol. 8, no. 3, pp. 591-605, 2015.

[8] Z. P. Fan, X. Zhang, F. D. Chen, and Y. Liu, "Multiple attribute decision making considering aspiration-levels: a method based on prospect theory," Computers \& Industrial Engineering, vol. 65, pp. 341-350, 2013.

[9] H. Hansson and C. J. Lagerkvist, "Decision making for animal health and welfare: integrating risk-benefit analysis with prospect theory," Risk Analysis, vol. 34, no. 6, pp. 1149-1159, 2014.

[10] Y. Dong, N. Luo, and H. Liang, "Consensus building in multiperson decision making with heterogeneous preference representation structures: a perspective based on prospect theory," Applied Soft Computing Journal, vol. 35, pp. 898-910, 2015.

[11] X. H. Li and X. H. Chen, "Extension of the TOPSIS method based on prospect theory and trapezoidal intuitionistic fuzzy numbers for group decision making," Journal of Systems Science and Systems Engineering, vol. 23, no. 2, pp. 231-247, 2014.

[12] N. Zhang, Z. Fang, and X. Liu, "Grey situation group decisionmaking method based on prospect theory," The Scientific World Journal, vol. 2014, Article ID 703597, 7 pages, 2014.

[13] C. L. Hwang and K. Yoon, Multiple Attribute Decision Making: Methods and Applications, Springer, New York, NY, USA, 1981.

[14] S. Opricovic and G. H. Tzeng, "Compromise solution by MCDM methods: a comparative analysis of VIKOR and TOPSIS," European Journal of Operational Research, vol. 156, no. 2, pp. 445-455, 2004.

[15] L. A. Zadeh, "Fuzzy sets," Information and Control, vol. 8, no. 3, pp. 338-353, 1965.

[16] F. Chiclana, F. Herrera, and E. Herrera-Viedma, "Integrating three representation models in fuzzy multipurpose decision making based on fuzzy preference relations," Fuzzy Sets and Systems, vol. 97, no. 1, pp. 33-48, 1998.

[17] H. Behret, "Group decision making with intuitionistic fuzzy preference relations," Knowledge-Based Systems, vol. 70, pp. 3343, 2014.

[18] H. Liao, Z. Xu, X.-J. Zeng, and D.-L. Xu, "An enhanced consensus reaching process in group decision making with intuitionistic fuzzy preference relations," Information Sciences, vol. 329, pp. 274-286, 2016.

[19] X. D. Peng and Y. Yang, "Algorithms for interval-valued fuzzy soft sets in stochastic multi-criteria decision making based on regret theory and prospect theory with combined weight," Applied Soft Computing, vol. 54, pp. 1-16, 2016. 
[20] X. Yang, T. Y. Lin, J. Yang, Y. Li, and D. Yu, "Combination of interval-valued fuzzy set and soft set," Computers \& Mathematics with Applications, vol. 58, no. 3, pp. 521-527, 2009.

[21] D. F. Li, "Multiattribute decision making models and methods using intuitionistic fuzzy sets," Journal of Computer \& System Sciences, vol. 70, no. 1, pp. 73-85, 2005.

[22] F. Q. Wang, X. Li, and X. H. Chen, "Hesitant fuzzy soft set and its applications in multicriteria decision making," Journal of Applied Mathematics, vol. 2014, Article ID 643785, 10 pages, 2014.

[23] L. I. De-Yi, C. Y. Liu, D. U. Yi, and X. Han, "Artificial intelligence with uncertainty," Journal of Software, vol. 15, no. 11, article 2, 2004.

[24] L. Deyi, M. Haijun, and S. Xuemei, "Membership clouds and membership cloud generators," Journal of Computer Research and Development, vol. 32, no. 6, pp. 15-20, 1995.

[25] W. Shuliang, L. Deren, S. Wenzhong, L. Deyi, and W. Xinzhou, "Cloud model-based spatial data mining," Geographical Information Science, vol. 9, no. 2, pp. 67-78, 2003.

[26] Y. Weng and Z. Zhongying, "Pattern mining for time series based on cloud theory pan-concept-tree," in Proceedings of the International Conference on Rough Sets and Current Trends in Computing, 2004.

[27] Z. Yunfang, D. Chaohua, and C. Weirong, "Adaptive probabilities of crossover and mutation in genetic algorithms based on cloud generators," Journal of Computational Information Systems, vol. 1, no. 4, pp. 671-678, 2005.

[28] P. Lv, L. Yuan, and J. Zhang, "Cloud theory-based simulated annealing algorithm and application," Engineering Applications of Artificial Intelligence, vol. 22, no. 4-5, pp. 742-749, 2009.

[29] D. Li, C. Liu, and W. Gan, "A new cognitive model: cloud model," International Journal of Intelligent Systems, vol. 24, no. 3, pp. 357-375, 2009.

[30] L. Y. Zhang, T. Li, and X. H. Xu, "Consensus model for multiple criteria group decision making under intuitionistic fuzzy environment," Knowledge-Based Systems, vol. 57, pp. 127135, 2014.

[31] F. Meng and X. Chen, "An approach to uncertain linguistic multi-attribute group decision making based on interactive index," International Journal of Uncertainty, Fuzziness and Knowledge-Based Systems, vol. 23, no. 3, pp. 319-344, 2015.

[32] P. Liu, F. Jin, X. Zhang, Y. Su, and M. Wang, "Research on the multi-attribute decision-making under risk with interval probability based on prospect theory and the uncertain linguistic variables," Knowledge-Based Systems, vol. 24, no. 4, pp. 554-561, 2011.

[33] S. L. Wang, Spatial data mining and knowledge discovery based on data field and cloud model [dissertation], Wuhan University, 2002.

[34] Y. J. Wang and H. S. Lee, "Generalizing TOPSIS for fuzzy multiple-criteria group decision-making," Computers \& Mathematics with Applications, vol. 53, no. 11, pp. 1762-1772, 2007.

[35] J. Ren, "Linguistic-stochastic multi-criterion decision-making method based on cloud model," Computer Integrated Manufacturing Systems, vol. 18, no. 12, pp. 2792-2797, 2012.

[36] J.-Q. Wang, P. Lu, H.-Y. Zhang, and X.-H. Chen, "Method of multi-criteria group decision-making based on cloud aggregation operators with linguistic information," Information Sciences, vol. 274, pp. 177-191, 2014.

[37] J.-Q. Wang, J.-J. Peng, H.-Y. Zhang, T. Liu, and X.-H. Chen, "An uncertain linguistic multi-criteria group decision-making method based on a cloud model," Group Decision and Negotiation, vol. 24, no. 1, pp. 171-192, 2015.

[38] K. Zhao, J. W. Gao, Z. Q. Qi, and C. B. Li, "Multi-criteria riskydecision-making approach based on prospect theory and cloud model," Control and Decision, vol. 30, no. 3, pp. 395-402, 2015.

[39] A. Tversky and D. Kahneman, "Advances in prospect theory: cumulative representation of uncertainty," Journal of Risk and Uncertainty, vol. 5, no. 4, pp. 297-323, 1992.

[40] J. Kacprzyk and M. Fedrizzi, "A 'soft' measure of consensus in the setting of partial (fuzzy) preferences," European Journal of Operational Research, vol. 34, no. 3, pp. 316-325, 1988.

[41] X. Chen, H. Zhang, and Y. Dong, "The fusion process with heterogeneous preference structures in group decision making: a survey," Information Fusion, vol. 24, pp. 72-83, 2015.

[42] D. E. Bell, "Regret in decision making under uncertainty," Operations Research, vol. 30, pp. 961-981, 1982.

[43] G. Loomes and R. Sugden, "Regret theory: an alternative theory of rational choice under uncertainty," The Economic Journal, vol. 92, no. 368, pp. 805-824, 1982.

[44] A. Muermann, O. S. Mitchell, and J. M. Volkman, "Regret, portfolio choice, and guarantees in defined contribution schemes," Insurance: Mathematics \& Economics, vol. 39, no. 2, pp. 219-229, 2006.

[45] S. Michenaud and B. Solnik, "Applying regret theory to investment choices: currency hedging decisions," Journal of International Money and Finance, vol. 27, no. 5, pp. 677-694, 2008. 


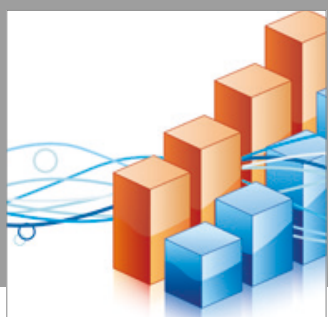

Advances in

Operations Research

vatersals

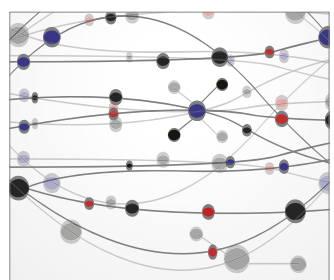

\section{The Scientific} World Journal
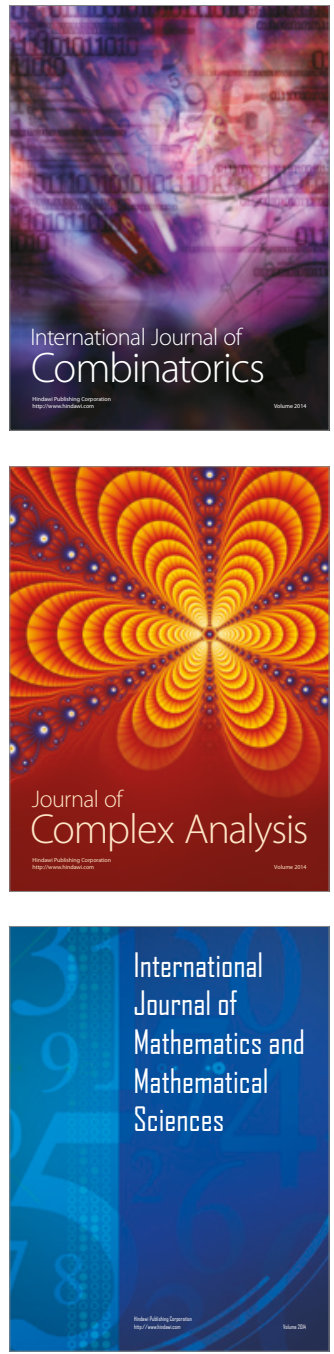
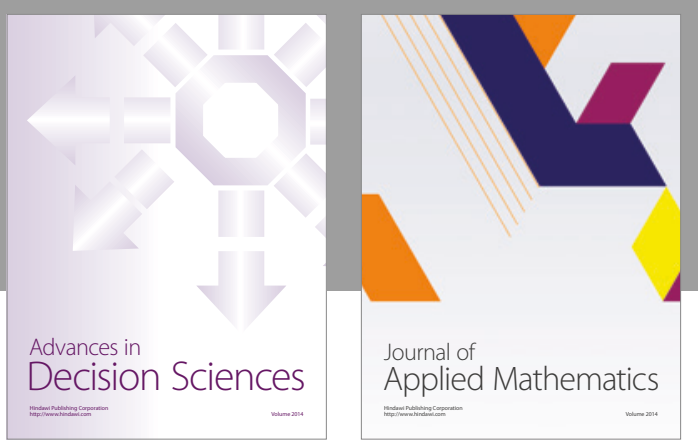

Algebra

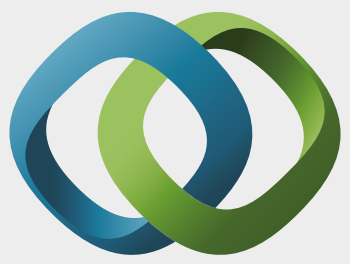

\section{Hindawi}

Submit your manuscripts at

https://www.hindawi.com
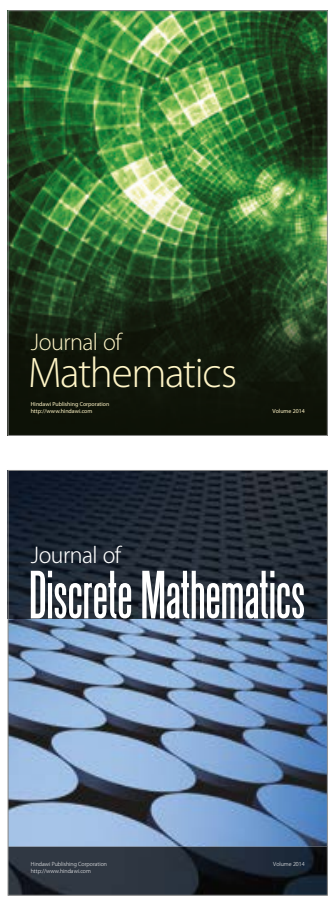

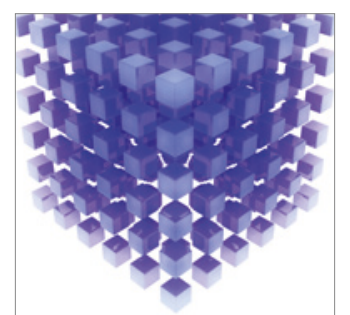

Mathematical Problems in Engineering
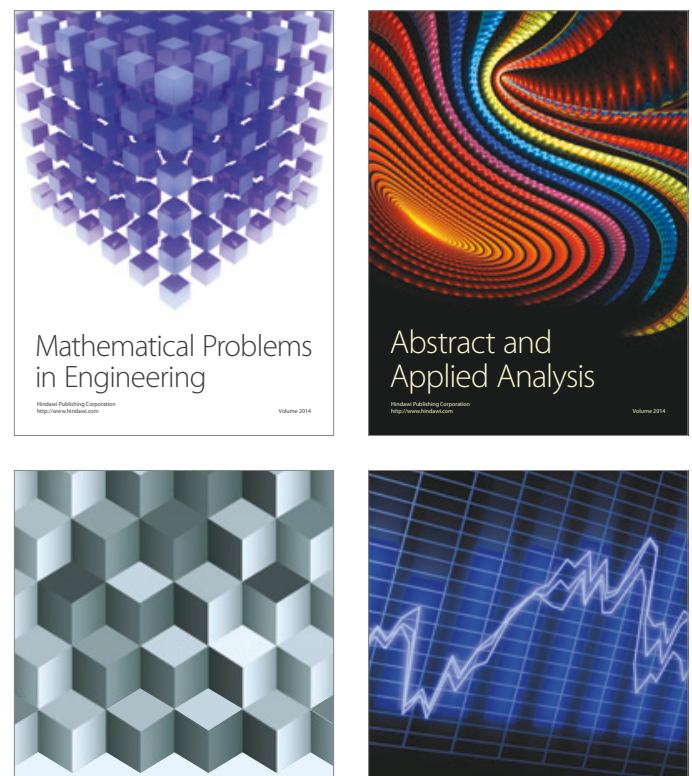

Journal of

Function Spaces

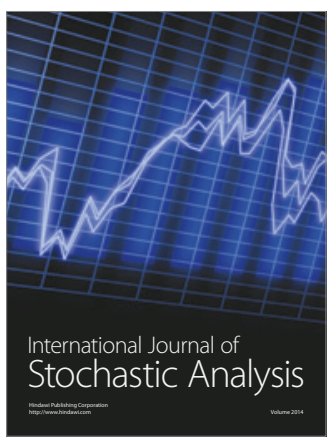

Probability and Statistics
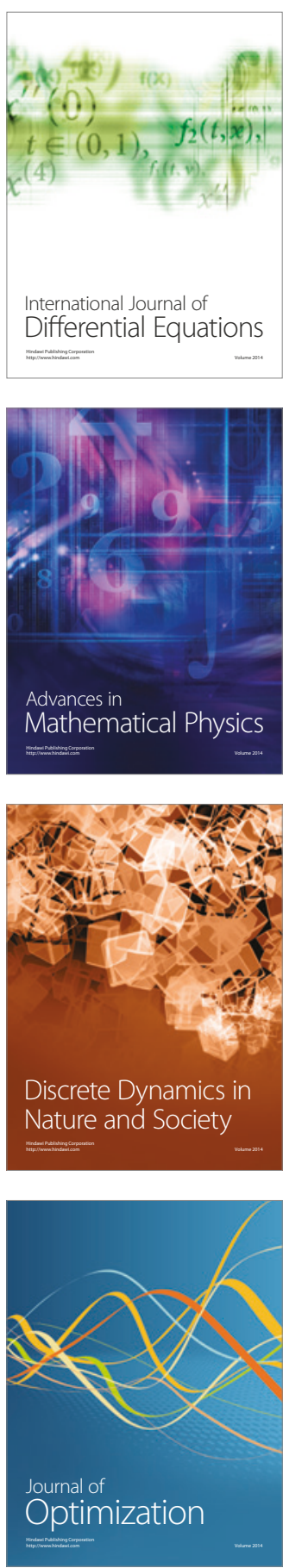